\title{
KIRWAN MAP AND MODULI SPACE OF FLAT CONNECTIONS
}

\author{
SÉBASTIEN RACANIÈRE
}

\begin{abstract}
If $K$ is a compact Lie group and $g \geq 2$ an integer, the space $K^{2 g}$ is endowed with the structure of a Hamiltonian space with a Lie group valued moment map $\Phi$. Let $\beta$ be in the centre of $K$. The reduction $\Phi^{-1}(\beta) / K$ is homeomorphic to a moduli space of flat connections. When $K$ is simply connected, a direct consequence of a recent paper of Bott, Tolman and Weitsman is to give a set of generators for the $K$-equivariant cohomology of $\Phi^{-1}(\beta)$.

Another method to construct classes in $H_{K}^{*}\left(\Phi^{-1}(\beta)\right)$ is by using the so called universal bundle. When the group is $\mathbf{S U}(n)$ and $\beta$ is a generator of the centre, these last classes are known to also generate the equivariant cohomology of $\Phi^{-1}(\beta)$. The aim of this paper is to compare the classes constructed using the result of Bott, Tolman and Weitsman and the ones using the universal bundle.

In particular, I prove that the set of cohomology classes coming from the universal bundle is indeed a set of multiplicative generators for the cohomology of the moduli space. With $K=\mathbf{S U}(n)$, this is a new proof of the well-known construction of generators for the cohomology of the moduli space of semi-stable vector bundles with fixed determinant.
\end{abstract}

\section{Index of notation}

$\mathbb{Z} \quad$ Group of relative integers

$\mathbb{R} \quad$ Field of reals

$g \quad$ Integer bigger than 1

$\mathbf{F} \quad$ Free group on $2 g$ generators $x_{1}, \ldots, x_{2 g}$

$R \quad$ Element in $\mathbf{F}$ given by $\prod_{j=1}^{g}\left[x_{2 j-1}, x_{2 j}\right]$

$\boldsymbol{\Pi} \quad$ Quotient of $\mathbf{F}$ by the relation $\prod_{j=1}^{g}\left[x_{2 j-1}, x_{2 j}\right]=1$

$\Sigma \quad$ Closed Riemann surface of genus $g$

$\Sigma_{0} \quad \Sigma$ with the interior of a disc removed

$K \quad$ Simply connected compact Lie group

$Z(K)$ Centre of $K$

$K_{c} \quad$ Quotient of $K$ by its centre $Z(K)$

\section{Introduction}

All cohomology will have coefficients in $\mathbb{R}$.

Let $\Sigma$ be a closed compact Riemann surface of genus $g$. Let $\Sigma_{0}$ be a compact Riemann surface with boundary obtained from $\Sigma$ by removing a small disc.

Received December 29, 2003.

2000 Mathematics Subject Classification. 53D20. 
Let $K$ be a compact simply connected Lie group. I denote by $K_{c}$ the quotient of $K$ by its centre $Z(K)$. In other words, $K_{c}$ is the projectivisation of $K$. Let $\beta$ be in the centre of $K$. Let $K$ act on itself by conjugation and on $K^{2 g}$ by diagonal conjugation. The point $\beta$ is fixed by $K$. Let $E K \longrightarrow B K$ be a classifying bundle for $K$. If $M$ is a topological space acted on by $K$, the equivariant cohomology $H_{K}^{*}(M)$ of $M$ is the singular cohomology of $M_{K}=M \times_{K} E K$. If $M$ is a manifold and it is acted on smoothly by $K$, then $H_{K}^{*}(M)$ is also the cohomology of the Cartan-de Rham complex $\Omega_{K}^{*}(M)$.

Let $\Phi$ be the equivariant map

$$
\begin{array}{ccc}
K^{2 g} & K \\
\left(X_{1}, \ldots, X_{2 g}\right) & \longmapsto & \prod_{i=1}^{g}\left[X_{2 i-1}, X_{2 i}\right] .
\end{array}
$$

Let $Y_{\beta}=\Phi^{-1}(\beta)$. The space $\Phi^{-1}(\beta) / K$ is homeomorphic to the moduli space of flat connections on the trivial bundle $K \times \Sigma_{0} \longrightarrow \Sigma_{0}$ (all $K$ principal bundles over $\Sigma_{0}$ are trivial) with prescribed holonomy $\beta$ around the boundary $S^{1}$ of $\Sigma_{0}$.

Call $\kappa$ the restriction map

$$
\kappa: H_{K}^{*}\left(K^{2 g}\right) \longrightarrow H_{K}^{*}\left(Y_{\beta}\right) .
$$

Let $b_{1}, \ldots, b_{r}$ be primitive elements which generate $H^{*}(K)$ and let $c_{1}, \ldots, c_{r}$ be their respective transgressions in $H^{*}(B K)$. One can extend $b_{1}, \ldots, b_{r}$ to equivariant classes $b_{1}^{K}, \ldots, b_{r}^{K}$ on $K$. Let $p r_{i}: K^{2 g} \longrightarrow K$ be the projection on the $i$-th factor and $b_{j, i}^{K}$ the pull-back of $b_{j}^{K}$ by $p r_{i}$.

Because $\Phi$ is a moment map on the quasi-Hamiltonian space $K^{2 g}$ (see [2]), the paper [5] gives a way of constructing equivariant classes $a_{1}, \ldots, a_{r}$ on $Y_{\beta}$ (see Equation (1) on page 424) such that $\left\{\kappa\left(c_{j}\right), \kappa\left(b_{j, i}^{K}\right), a_{j}\right.$, $j=1, \ldots, r, i=1, \ldots, 2 g\}$ generate the $K$-equivariant cohomology of $Y_{\beta}$.

Also, one can construct a $K$-equivariant $K_{c}$-principal bundle over $\Sigma \times Y_{\beta}$ : the so called universal bundle. The Künneth decomposition of the equivariant characteristic classes of this bundle allows me to construct classes $\left\{c_{j}^{\prime}, b_{j, i}^{\prime}, a_{j}^{\prime}, j=\right.$ $1, \ldots, r, i=1, \ldots, 2 g\}$ in $H_{K}^{*}\left(Y_{\beta}\right)$ (see Equation (2) on page 425). At least when $K$ is the special unitary group and $\beta$ is a generator of the centre of $K$, these classes are known to generate $H_{K}^{*}\left(Y_{\beta}\right)$ as a ring. They are the Atiyah-BottBiswas-Raghavendra classes ${ }^{1}$.

The aim of this paper is to prove the following Theorem.

Theorem 3.2. Let $K$ be a compact, connected and simply connected Lie group. Let $\beta$ be in the centre of $K$ such that $K$ acts locally freely on $Y_{\beta}$. Let

\footnotetext{
${ }^{1}$ Atiyah and Bott [1] were the first ones to construct generators of the cohomology of the moduli space. Their classes depended on the choice of a universal bundle which was only defined up to the tensor product with a line bundle. The universal bundle of this article is actually the projectivisation of theirs. It is well-defined and so are the generators constructed from it. This was proved by Biswas and Raghavendra in [3]
} 
$a_{j}^{\prime}, b_{j, i}^{\prime}, c_{j}^{\prime}, a_{j}, b_{j, i}^{K}, c_{j}$ be equivariant classes defined as above. The following relations hold

$$
\begin{aligned}
c_{j}^{\prime} & =\kappa\left(c_{j}\right) \\
b_{j, i}^{\prime} & =\kappa\left(b_{j, i}^{K}\right) .
\end{aligned}
$$

Also, the classes $\left\{a_{j}\right\}$ depend on certain choices but one can choose them so that

$$
a_{j}^{\prime}=a_{j}
$$

This article is organised as followed. In Section 2, I recall what [5] says about the restriction map involved in the reduction of $K^{2 g}$ at $\beta$. I also show how to use this result to construct generators for the $K$-equivariant cohomology of $Y_{\beta}$. In Section 3, I describe the construction of equivariant characteristic classes on $Y_{\beta}$ using a universal bundle over $\Sigma \times Y_{\beta}$. I also outline the proof of Theorem 3.2, leaving the most technical details for Section 4.

\section{Generators for the equivariant cohomology of $Y_{\beta}$}

Let $\boldsymbol{\Pi}$ be the fundamental group of $\Sigma$. Fix a presentation

$$
\boldsymbol{\Pi}=\left\langle x_{1}, \ldots, x_{2 g} ; \prod_{j=1}^{g}\left[x_{2 j-1}, x_{2 j}\right]=1\right\rangle .
$$

Let $\mathbf{F}$ be the fundamental group of $\Sigma_{0}$. Fix a presentation

$$
\mathbf{F}=\left\langle x_{1}, \ldots, x_{2 g}\right\rangle
$$

such that the map

$$
\mathbf{F} \longrightarrow \boldsymbol{\Pi}
$$

induced by the inclusion of $\Sigma_{0}$ in $\Sigma$, is given by the obvious map $x_{k} \longmapsto x_{k}$.

Let $\beta$ be in the centre of $K$. Let $Y_{\beta}$ be the subset of $K^{2 g}$ defined by

$$
Y_{\beta}=\left\{\left(X_{1}, \ldots, X_{2 g}\right) \in K^{2 g} \mid \prod_{j=1}^{g}\left[X_{2 j-1}, X_{2 j}\right]=\beta\right\} .
$$

The group $K$ acts on $K^{2 g}$ by conjugation. This action restricts to $Y_{\beta}$, since $\beta$ is in the centre of $K$. The quotient $\mathcal{M}_{\beta}=Y_{\beta} / K$ can be identified with a moduli space of flat connections on the trivial principal bundle $K \times \Sigma_{0} \longrightarrow \Sigma_{0}$ (because $K$ is simply connected, all principal bundles are trivial over $\Sigma_{0}$ ), with holonomy $\beta$ around the boundary $\partial \Sigma_{0}$. If $K$ acts locally freely on $Y_{\beta}$, then the cohomology of $\mathcal{M}_{\beta}$ is isomorphic to the $K$-equivariant cohomology of $Y_{\beta}$ (recall that cohomology is taken with coefficients in $\mathbb{R}$ ).

Let $\kappa$ be the restriction map

$$
\kappa: H_{K}^{*}\left(K^{2 g}\right) \longrightarrow H_{K}^{*}\left(Y_{\beta}\right) .
$$

The space $K^{2 g}$ has the structure of a quasi-Hamiltonian space (see [2]) with moment map

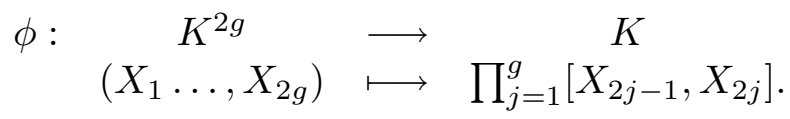


The map $\phi$ is a submersion at any point of $Y_{\beta}=\phi^{-1}(\beta)$ if and only if $K$ acts locally freely on $Y_{\beta}$. In this nice case, the space $\mathcal{M}_{\beta}$ is a compact symplectic orbifold since it is the regular reduction of $K^{2 g}$ at $\beta$.

Let $b_{1}, \ldots, b_{r}$ be primitive elements in $H^{*}(K)$ that generate the cohomology of $K$ as a ring

$$
H^{*}(K)=\bigwedge\left(\sum_{j=1}^{r} \mathbb{R} b_{j}\right) .
$$

Each $b_{j}$ is of odd degree.

Let $c_{1}, \ldots, c_{r}$ be the transgressions in $H^{*}(B K)$ of respectively $b_{1}, \ldots, b_{r}$, thus $\operatorname{deg} c_{j}=\operatorname{deg} b_{j}+1$ and

$$
H^{*}(B K)=\mathbb{R}\left[c_{1}, \ldots, c_{r}\right] .
$$

For each $K$-principal bundle $G \longrightarrow X$, the classes $c_{1}, \ldots, c_{r}$ define characteristic classes $c_{1}(G), \ldots, c_{r}(G)$ in $H^{*}(X)$.

Proposition 2.1. Let $E K \longrightarrow B K$ be the classifying bundle for $K$. The bundle $E K \times_{K} K^{n} \longrightarrow B K$ is cohomologically trivial as a ring, that is

$$
H_{K}^{*}\left(K^{n}\right) \simeq H^{*}(B K) \otimes H^{*}\left(K^{n}\right)
$$

as rings.

This result is not new. I propose here a proof which is original as far as I know.

Also, because I want to state the principal results as soon as possible, I will make use in the following proof, as well as in others, of Lemma 3.5 even if I haven't proved it yet.

Proof. I will prove the case $n=1$. The general case is similar, one only has to use Proposition 3.6 instead of Lemma 3.5 (or use the fact that if $X$ has an equivariantly formal action of $K$ then the diagonal action of $K$ on $X^{n}$ is also equivariantly formal).

Let me first prove that $H_{K}^{*}(K)$ and $H^{*}(B K) \otimes H^{*}(K)$ are isomorphic as $H^{*}(B K)$ modules. By the Leray-Hirsch Theorem, all I have to do is prove that the homomorphism

$$
H_{K}^{*}(K) \longrightarrow H^{*}(K)
$$

is surjective. Let $b_{j}$ be one of the generators of $H^{*}(K)$. Let $D \longrightarrow S^{1} \times K$ be the $K$-equivariant $K$-principal bundle of Lemma 3.5. Its total space $D$ is $(\mathbb{R} \times K \times K) / \mathbb{Z}$ where the action of $\mathbb{Z}$ is defined by

$$
\begin{array}{ccc}
\mathbb{Z} \times(\mathbb{R} \times K \times K) & \longrightarrow & \mathbb{R} \times K \times K \\
\left(\lambda, t, k_{1}, k_{2}\right) & \longmapsto & \left(t+\lambda, k_{1}, k_{1}^{\lambda} k_{2}\right)
\end{array}
$$

and the projection is

$$
\begin{array}{ccc}
(\mathbb{R} \times K \times K) / \mathbb{Z} & \longrightarrow & S^{1} \times K \\
\left\{t, k_{1}, k_{2}\right\} & \longmapsto & \left(\{t\}, k_{1}\right) .
\end{array}
$$

As a $K$-principal bundle, its characteristic class $c_{j}(D)$ is $\mathrm{d} t \otimes b_{j}$ (Lemma 3.5). As an immediate corollary, I deduce that there exists $b_{j}^{K}$ in $H_{K}^{*}(K)$ such that 
$c_{j}\left(D \times_{K} E K\right)=1 \otimes c_{j}+\mathrm{d} t \otimes b_{j}^{K}$, where $b_{j}^{K}$ restricts to $b_{j}$. Now, because the $b_{j}$ 's generate $H^{*}(K)$ as a ring, the homomorphism

$$
H_{K}^{*}(K) \longrightarrow H^{*}(K)
$$

is surjective and I have proved the existence of a $H^{*}(B K)$ module isomorphism between $H_{K}^{*}(K)$ and $H^{*}(B K) \otimes H^{*}(K)$. The existence of a ring isomorphism follows because the cohomology $H^{*}(K)$ of the fibre in $K \times_{K} E K \longrightarrow B K$ is generated by classes of odd degree (see [11]).

Remark 2.2. Let $s$ be the section

$$
\begin{array}{ccc}
s: \quad B K & \longrightarrow & E K \times_{K} K \\
{[u]} & \longmapsto & {[u, 1] .}
\end{array}
$$

Via id $\times s: S^{1} \times B K \longrightarrow S^{1} \times K_{K}$, the bundle $D_{K} \longrightarrow S^{1} \times K_{K}$ pulls back to $S^{1} \times E K \longrightarrow S^{1} \times B K$. Hence $s^{*} b_{j}^{K}=0$.

Let $n$ be an integer (I will need it to be either 1 or $2 g$ ). Let $b_{j, i}^{K}$ be the pull-back, under the projection on the $i$-th factor $K^{n} \longrightarrow K$, of $b_{j}^{K}$.

The following Lemma is a consequence of Proposition 2.1.

Lemma 2.3. The $K$-equivariant cohomology of $K^{n}$ is isomorphic as a ring to

$$
H_{K}^{*}\left(K^{n}\right) \simeq \bigwedge\left(\sum \mathbb{R} b_{j, i}^{K}\right) \otimes \mathbb{R}\left[c_{1}, \ldots, c_{r}\right] .
$$

Before stating the result of Bott, Tolman and Weitsman for $\kappa$, I need to construct some classes in $H_{K}^{*}\left(Y_{\beta}\right)$.

Lemma 2.4. For all $j$, the pull-back under $\phi^{*}$ of $b_{j}^{K}$ is zero

$$
\phi^{*}\left(b_{j}^{K}\right)=0 .
$$

In their article [5, Remark 2.3], the authors already gave this result. I give here a proof using Lemma 3.5.

Proof. Consider the diagram

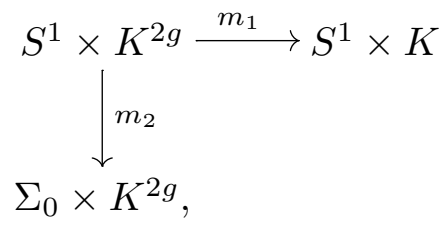

where the horizontal map $m_{1}$ is induced by the moment map $\phi: K^{2 g} \longrightarrow K$ and the vertical map $m_{2}$ is given by the injection of $S^{1}$ as the boundary of $\Sigma_{0}$. The pull-back of $D$ by $m_{1}$ and the pull-back of $F$ by $m_{2}$ are isomorphic. Indeed they are both equivariantly isomorphic to the bundle

$$
\left(\mathbb{R} \times K^{2 g} \times K\right) / \mathbb{Z} \longrightarrow S^{1} \times K^{2 g}
$$

where the group $\mathbb{Z}$ acts by

$$
\begin{array}{ccc}
\mathbb{Z} \times \mathbb{R} \times K^{2 g} \times K & \longrightarrow & \mathbb{R} \times K^{2 g} \times K \\
\left(\lambda, t,\left(X_{1}, \ldots, X_{2 g}\right), k\right) & \longmapsto & \left(t+\lambda,\left(X_{1}, \ldots, X_{2 g}\right),\left(\prod_{j=1}^{g}\left[X_{2 j-1}, X_{2 j}\right]\right)^{\lambda} k\right) .
\end{array}
$$


One then deduces the Lemma from Proposition 3.6 and Lemma 3.5 by noticing that the injection of $S^{1}$ as the boundary of $\Sigma_{0}$ induces the null map in degree 1 cohomology.

Let $\overline{b_{1}}, \ldots, \overline{b_{r}}$ be equivariant forms representing respectively $b_{1}^{K}, \ldots, b_{r}^{K}$.

Remark 2.5. Such forms will be chosen in Section 4 so that their pull-backs by the section s of Remark 2.2 vanish. This will be proved in Section 4.

The preceding Lemma implies there exist equivariant forms $\overline{a_{1}}, \ldots, \overline{a_{r}}$ on $K^{2 g}$ such that

$$
\phi^{*}\left(\overline{b_{j}}\right)=\mathrm{d} \overline{a_{j}}, \forall j .
$$

The $\overline{a_{1}}, \ldots, \overline{a_{r}}$ are closed when restricted to $Y_{\beta}$. They define classes $a_{1}, \ldots, a_{r}$ in $H_{K}^{*}\left(Y_{\beta}\right)$.

Theorem 2.6 ( Bott, Tolman and Weitsman ). The equivariant cohomology $H_{K}^{*}\left(Y_{\beta}\right)$ is generated as a ring by the image of $\kappa$ and the $a_{1}, \ldots, a_{r}$.

The following Corollary is an immediate consequence.

Corollary 2.7. The equivariant cohomology $H_{K}^{*}\left(Y_{\beta}\right)$ is generated as a ring by the classes $\kappa\left(c_{j}\right), \kappa\left(b_{j, i}^{K}\right), a_{j}$ for $j=1, \ldots, r$ and $i=1, \ldots, 2 g$.

\section{Some classical classes in $H_{K}^{*}\left(Y_{\beta}\right)$}

Let $\widetilde{\Sigma}$ be the universal cover of $\Sigma$. The group $\Pi$ acts on $\widetilde{\Sigma}$. Since I have chosen a presentation for $\mathbf{F}$, there is a preferred isomorphism

$$
\begin{array}{clc}
\operatorname{Hom}(\mathbf{F}, K) & \longrightarrow & K^{2 g} \\
\rho & \longmapsto & \left(\rho\left(x_{1}\right), \ldots, \rho\left(x_{2 g}\right)\right) .
\end{array}
$$

I will use this isomorphism to identify $\operatorname{Hom}(\mathbf{F}, K)$ and $K^{2 g}$. Define an action of П on $\widetilde{\Sigma} \times Y_{\beta} \times K_{c}$

$$
\begin{array}{ccc}
\Pi \times\left(\widetilde{\Sigma} \times Y_{\beta} \times K_{c}\right) & \longrightarrow & \widetilde{\Sigma} \times Y_{\beta} \times K_{c} \\
(p, \sigma, \rho, k) & \longmapsto & (p \sigma, \rho, \rho(\tilde{p}) k),
\end{array}
$$

where $\tilde{p}$ is any element in $\mathbf{F}$ lying above $p$. This action is well-defined and free. The quotient $E$, together with the projection $\pi: E \longrightarrow \Sigma \times Y_{\beta}$ define a $K_{c}$-principal bundle. The actions of $K$ on $Y_{\beta}$ by conjugation and on $K_{c}$ by multiplication on the left, make $E$ into a $K$-equivariant $K_{c}$-principal bundle over $\Sigma \times Y_{\beta}$. I call it the universal bundle (this bundle is also constructed in Jeffrey [9]).

Example 3.1. For $K=\mathbf{S U}(n)$ and $\beta$ a generator of $Z(\mathbf{S U}(n))$, this is the projectivisation of a universal bundle for the moduli space of semi-stable vector bundles of fixed rank and determinant (see [1] and [11]).

Because $K$ is compact and simply connected, I know that

$$
H^{*}(B K) \simeq H^{*}\left(B K_{c}\right) \text {. }
$$


The classes $c_{1}, \ldots, c_{r}$ in $H^{*}\left(B K_{c}\right)$ define equivariant characteristic classes for the bundle $E$ (recall that these are the same as the usual characteristic classes of the $K$-principal bundle $\left.E \times_{K} E K \longrightarrow \Sigma \times Y_{\beta} \times_{K} E K\right)$. These classes live in $H_{K}^{*}\left(\Sigma \times Y_{\beta}\right) \simeq H^{*}(\Sigma) \otimes H_{K}^{*}\left(Y_{\beta}\right)$. Let $\alpha_{1}, \ldots, \alpha_{2 g}$ be a basis of $H^{*}(\Sigma)$, dual to $x_{1}, \ldots, x_{2 g}$ in $\boldsymbol{\Pi}=\pi_{1}(\Sigma)$. Let $\mathcal{V}$ be a volume form of volume 1 on $\Sigma$. Using the Künneth decomposition, write

$$
c_{j}\left(E \times_{K} E K\right)=1 \otimes c_{j}^{\prime}+\sum_{i=1}^{2 g} \alpha_{i} \otimes b_{j, i}^{\prime}+\nu \otimes a_{j}^{\prime} .
$$

In Example 3.1, these classes are the known Atiyah-Bott - Biswas-Raghavendra generators of $H_{K}^{*}\left(Y_{\beta}\right)$ (see [1] and [3]). A natural question is: what is the relation between these classes and the ones of Corollary 2.7? The answer is given in the following Theorem.

Theorem 3.2. Let $K$ be compact, connected and simply connected Lie group. Let $\beta$ be in the centre of $K$. Assume that $\beta$ is a regular value of the moment map $\Phi$. Let the $\left\{a_{j}^{\prime}, b_{j, i}^{\prime}, c_{j}^{\prime}, a_{j}, b_{j, i}^{K}, c_{j}\right\}$ be equivariant classes defined as above. The following relations hold

$$
\begin{aligned}
c_{j}^{\prime} & =\kappa\left(c_{j}\right) \\
b_{j, i}^{\prime} & =\kappa\left(b_{j, i}^{K}\right) .
\end{aligned}
$$

Also, the classes $\left\{a_{j}\right\}$ depend on certain choices but one can choose them so that

$$
a_{j}^{\prime}=a_{j}
$$

Proof. Fix $j$. I will start by proving the first part of the Theorem, that is I will compute the $\left\{c_{j}^{\prime}, b_{j, i}^{\prime}\right\}$.

Let $\widetilde{\Sigma}_{0}$ be the universal covering for $\Sigma_{0}$. Define an action of $\mathbf{F}=\pi_{1}\left(\Sigma_{0}\right)$ on $\widetilde{\Sigma}_{0} \times K^{2 g} \times K$

$$
\begin{array}{ccc}
\mathbf{F} \times \widetilde{\Sigma}_{0} \times K^{2 g} \times K & \longrightarrow & \widetilde{\Sigma}_{0} \times K^{2 g} \times K \\
(p, \sigma, \rho, k) & \longmapsto & (p \sigma, \rho, \rho(p) k) .
\end{array}
$$

This action is free and the quotient $F$ is a $K$-principal bundle on $\Sigma_{0} \times K^{2 g}$. The action of $K$ by conjugation on $K^{2 g}$ and by multiplication on the left on $K$ makes $F$ into a $K$-equivariant $K$-principal bundle. The situation here is somehow confusing since there are two different actions of $K$ on $F$. In order to clarify the situation, I will call the action that makes $F$ a $K$-principal bundle the principal action and call the action that makes it $K$-equivariant the covering action ('covering' because it covers the action of $K$ on $K^{2 g}$ ).

Proposition 3.3. The projectivisation of $\left.F\right|_{\Sigma_{0} \times Y_{\beta}}$ is $K$-equivariantly isomorphic to $\left.E\right|_{\Sigma_{0} \times Y_{\beta}}$.

Proof. The proof is easy. 
Call $D_{n}$ the restriction of $F$ to the $n$-th circle in the wedge product times the $n$-th copy of $K$ in $K^{2 g}$. I identify the circle $S^{1}$ with the quotient $\mathbb{R} / \mathbb{Z}$. I denote by $t$ the natural coordinate in $\mathbb{R}$, thus $d t$ defines a volume form on $S^{1}$.

Lemma 3.4. All the $D_{n}$ 's are isomorphic to the same bundle $D \longrightarrow S^{1} \times K$. The total space $D$ is $(\mathbb{R} \times K \times K) / \mathbb{Z}$ where the action of $\mathbb{Z}$ is defined by

$$
\begin{array}{ccc}
\mathbb{Z} \times(\mathbb{R} \times K \times K) & \longrightarrow & \mathbb{R} \times K \times K \\
\left(\lambda, t, k_{1}, k_{2}\right) & \longmapsto & \left(t+\lambda, k_{1}, k_{1}^{\lambda} k_{2}\right)
\end{array}
$$

and the projection is

$$
\begin{array}{ccc}
(\mathbb{R} \times K \times K) / \mathbb{Z} & \longrightarrow & S^{1} \times K \\
\left\{t, k_{1}, k_{2}\right\} & \longmapsto & \left(\{t\}, k_{1}\right) .
\end{array}
$$

Proof. Fix an integer $n$ between 1 and $2 g$ and let $x=x_{n}$ be the $n$-th generator of $\mathbf{F}$.

Recall that $F$ was defined as a quotient

$$
F=\left(\widetilde{\Sigma}_{0} \times K^{2 g} \times K\right) / \pi_{1}\left(\Sigma_{0}\right) .
$$

Let $h$ be the group homomorphism

$$
\begin{array}{ccc}
\mathbb{Z} & \longrightarrow & \pi_{1}\left(\Sigma_{0}\right) \\
\lambda & \longmapsto & x^{\lambda} .
\end{array}
$$

This morphism makes $\mathbb{Z}$ into a subgroup of $\pi_{1}\left(\Sigma_{0}\right)$. Choose a point $\widetilde{\sigma_{0}}$ in $\Sigma_{0}$ lying above the base point $\sigma_{0}$ of $\Sigma_{0}$. Choose a loop $S^{1} \longrightarrow \Sigma_{0}$ representing $x$. This loop lifts to a map $\chi: \mathbb{R} \longrightarrow \widetilde{\Sigma}_{0}$ such that $\chi(0)=\widetilde{\sigma_{0}}$ and for $\lambda$ an integer $\chi(t+\lambda)=x^{\lambda} \cdot \chi(t)$.

The covering action of $K$ (the one that covers the action of $K$ on $S^{1} \times K$ ) on $D_{n}$ is induced by the conjugation on the first factor $K$ in $\mathbb{R} \times K \times K$ and by multiplication on the left on the second factor $K$.

Let $\iota: K \longrightarrow K^{2 g}$ be the injection of the $n$-th factor. Define a map

$$
\begin{array}{ccc}
(\mathbb{R} \times K \times K) / \mathbb{Z} & \longrightarrow & \left(\widetilde{\Sigma}_{0} \times K^{2 g} \times K\right) / \pi_{1}\left(\Sigma_{0}\right) \\
{[t, X, k]} & \longmapsto & {[\chi(t), \iota(X), k] .}
\end{array}
$$

This map is well-defined since it sends the translation $\left[t+\lambda, X, X^{\lambda} k\right]$ of $[t, X, k]$ by $\lambda$ to

$$
\begin{aligned}
& {\left[\chi(t+\lambda), \iota(X), X^{\lambda} k\right] } \\
= & {\left[x_{n}^{\lambda} \cdot \chi(t), \iota(X), X^{\lambda} k\right] } \\
= & {[\chi(t), \iota(X), k] . }
\end{aligned}
$$

It is also $K$-equivariant because, for $\ell$ in $K$, it sends $\left[t, \operatorname{Ad}_{\ell} X, \ell k\right]$ to

$$
\begin{aligned}
& {\left[\chi(t), \iota\left(A d_{\ell} X\right), \ell k\right] } \\
= & {\left[\chi(t), A d_{\ell} \circ \iota(X), \ell k\right] } \\
= & \ell \cdot[\chi(t), \iota(X), k] .
\end{aligned}
$$

The Lemma follows. 
Lemma 3.5. The characteristic classes of $D$ are

$$
c_{j}(D)=d t \otimes b_{j}
$$

Proof. The proof is to be found in the next Section.

The previous Lemma is actually very useful since it can be used to prove Proposition 3.6 which leads to Theorem 3.2, and also Proposition 2.1 and Lemma 2.4 .

Now I wish to compute the equivariant characteristic classes of $F$. The cohomologies of $\Sigma$ and $\Sigma_{0}$ are the same in degree 0 and 1 and $\Sigma_{0}$ has no cohomology in degree 2. I use the same basis $\alpha_{1}, \ldots, \alpha_{2 g}$ for $H^{1}(\Sigma)$ and $H^{1}\left(\Sigma_{0}\right)$.

Proposition 3.6. Using the Künneth decomposition, the equivariant characteristic classes of $F$ are

$$
c_{j}\left(F \times_{K} E K\right)=1 \otimes c_{j}+\sum_{i=1}^{2 g} \alpha_{i} \otimes b_{j, i}^{K} .
$$

Proof. The surface $\Sigma_{0}$ is homotopic to a wedge product of $2 g$ circles. As is easily seen, Proposition 3.6 will follow from the computation of the characteristic classes of the restriction of $F$ to the $n$-th circle in the wedge product times the $m$-th copy of $K$ in $K^{2 g}$, that is $D_{n}$. When $n \neq m$, the restriction of $F$ is trivial. Thus, I only have to do the computation for $n=m$. This is given by Lemma 3.5.

The first part of the Theorem is a consequence of Proposition 3.3 and Proposition 3.6. Indeed, by Proposition 3.3

$$
\left.c_{j}\left(E \times_{K} E K\right)\right|_{\Sigma_{0} \times Y_{\beta}}=\left.c_{j}\left(F \times_{K} E K\right)\right|_{\Sigma_{0} \times Y_{\beta}} .
$$

But

$$
\left.c_{j}\left(E \times_{K} E K\right)\right|_{\Sigma_{0} \times Y_{\beta}}=1 \otimes c_{j}^{\prime}+\sum_{i=1}^{2 g} \alpha_{i} \otimes b_{j, i}^{\prime}
$$

and by Proposition 3.6

$$
\begin{aligned}
c_{j}\left(F \times_{K} E K\right) \mid \Sigma_{0} \times Y_{\beta} & =\left(\mathrm{id}^{*} \times \kappa\right) c_{j}\left(F \times_{K} E K\right) \\
& =1 \otimes \kappa\left(c_{j}\right)+\sum_{i=1}^{2 g} \alpha_{i} \otimes \kappa\left(b_{j, i}^{K}\right) .
\end{aligned}
$$

The first part of the Theorem follows by comparing Equation (3) and Equation (4).

The second part of Theorem 3.2 is a consequence of the following Lemma and of the definition of the classes $a_{1}, \ldots, a_{r}$. 
Lemma 3.7. One can choose representatives $\bar{b}_{j}^{K}$ and $\bar{a}_{j}^{\prime}$ of respectively $b_{j}^{K}$ and $a_{j}^{\prime}$ such that $\bar{a}_{j}^{\prime}$ extends to an equivariant class on $K^{2 g}$ whose differential is $\Phi^{*}\left(\bar{b}_{j}^{K}\right)$.

The proof of this Lemma, together with the proof of Lemma 3.5, is given in the next Section.

This finishes the proof of Theorem 3.2.

Remark 3.8. If the group $K$ is a torus $T$, then it is not simply connected and one can not apply Theorem 3.2 to find generators of the cohomology of the moduli space. Nevertheless, in this case the moduli space is either $T^{2 g}$ if $\beta=1$ or is empty otherwise. Its cohomology is thus very simple. In general, a connected compact Lie group is the quotient by a finite group of a product of a simply connected group and a torus. Combining what I have just said about tori with Theorem 3.2, one can deduce generators for the cohomology of the moduli space.

\section{Proof of Lemma 3.7, Remark 2.5 and Lemma 3.5}

The aim of this section is to prove Lemma 3.5, Remark 2.5 and Lemma 3.7.

The proof of Lemma 3.5 goes as follows: in a similar way to Jeffrey[9], I construct explicitly a map from $S^{1} \times K$ to the fat realisation of $B K$ (see [6] and [12]) as a simplicial manifold such that the pull-back of $E K$ under this map is the principal bundle $D_{n}$. I then use a result of Shulman to prove Lemma 3.5.

I will start by introducing the simplicial realisations of $E G$ and $B G$ for any group $G$ (see [12]). Let

$$
\Delta^{m}=\left\{\left(t_{0}, \ldots, t_{m}\right) \in[0,1]^{m+1} \mid \sum_{i=0}^{m} t_{i}=1\right\}
$$

be the standard $m$-simplex. The simplicial realisation $\overline{\mathrm{NG}}$, respectively $\mathrm{NG}$, of $E G$, respectively $B G$, is given by the contravariant functor

$$
\left\{\Delta^{m}, m \in \mathbb{N}\right\} \longrightarrow\{\text { category of smooth manifolds }
$$

which to each $\Delta_{m}$ associates $\overline{\mathrm{NG}}(m)=G^{m+1}$, respectively $\mathrm{NG}(m)=G^{m}$. For $i$ between 0 and $m$, the map $\bar{\epsilon}_{i}: \overline{\mathrm{NG}}(m) \longrightarrow \overline{\mathrm{NG}}(m-1)$, respectively $\epsilon_{i}$ : $\mathrm{NG}(m) \longrightarrow \mathrm{NG}(m-1)$, corresponding to the $i$-th face map $\epsilon^{i}: \Delta^{m-1} \longrightarrow \Delta^{m}$ is given by the omission of the $i$-th term

$$
\bar{\epsilon}_{i}\left(k_{0}, \ldots, k_{m}\right)=\left(k_{0}, \ldots, \check{k}_{i}, \ldots, k_{m}\right),
$$

respectively

$$
\epsilon_{i}\left(k_{1}, \ldots, k_{m}\right)= \begin{cases}\left(k_{2}, \ldots, k_{m}\right) & i=0 \\ \left(k_{1}, k_{2}, \ldots, k_{i} k_{i+1}, \ldots, k_{m}\right) & i=1, \ldots, m-1 \\ \left(k_{1}, \ldots, k_{m-1}\right) & i=m\end{cases}
$$

The projection $\overline{\mathrm{NG}}(m) \longrightarrow \mathrm{NG}(m)$ is the map

$$
q_{m}\left(k_{0}, \ldots, k_{m}\right)=\left(k_{1} k_{2}^{-1}, \ldots, k_{m-1} k_{m}^{-1}\right) .
$$


I then take $E G$, respectively $B G$, to be the fat realisation of $\overline{\mathrm{NG}}$, resp. NG, that is $E G$ is the quotient of $\bigsqcup_{m} \Delta^{m} \times \overline{\mathrm{NG}}(m)$ by the relation

$$
\left(\epsilon^{i} \times \mathrm{id}\right)(t, x) \sim\left(i d \times \bar{\epsilon}_{i}\right)(t x)
$$

for $(t, x)$ in $\Delta^{m-1} \times \overline{\mathrm{NG}}(m)$ and $B G$ is the quotient of $\bigsqcup_{m} \Delta^{m} \times \mathrm{NG}(m)$ by the relation

$$
\left(\epsilon^{i} \times \mathrm{id}\right)(t, x) \sim\left(i d \times \epsilon_{i}\right)(t x)
$$

for $(t, x)$ in $\Delta^{m-1} \times \mathrm{NG}(m)$. The projection $q: E G \longrightarrow B G$ is induced by the maps $q_{m}$. The action of $G$ on $E G$ is induced by the diagonal multiplication of $G$ on the right on $\overline{\mathrm{NG}}(m)=G^{m+1}$ for each $m$. In fact, there is a second action of $G$ on $E G$, the covering action, which descends to an action of $G$ on $B G$. This action on $E G$ is induced by the diagonal multiplication on the left of $G$ on $\overline{\mathrm{NG}}(m)=G^{m+1}$, it descends to an action on $B G$ induced by the diagonal conjugation on $\mathrm{NG}(m)=G^{m}$.

Notice that

$$
\begin{aligned}
S^{1} & =B \mathbb{Z} \\
\Sigma & =B \boldsymbol{\Pi} \\
\Sigma_{0} & =B \mathbf{F} .
\end{aligned}
$$

Proof of Lemma 3.5. In the simplicial models, the injection of $\Sigma_{0}$ in $\Sigma$ is given by the projections

$$
\mathbf{F}^{n} \longrightarrow \mathbf{\Pi}^{n} \text {. }
$$

The surface $\Sigma_{0}$ is homotopic to the wedge product of $2 g$ circles. The inclusion of the $i$-th circle $S^{1} \longrightarrow \Sigma_{0}$ is given by

$$
\begin{array}{ccc}
\mathbb{Z}^{n} & \longrightarrow \mathbf{F}^{n} \\
\lambda & \longmapsto & \left(x_{i}^{\lambda}, \ldots, x_{i}^{\lambda}\right) .
\end{array}
$$

Let $R=\prod_{j=1}^{g}\left[x_{2 j-1}, x_{2 j}\right]$ be in $\mathbf{F}$. The inclusion of $S^{1}$ as the boundary of $\Sigma_{0}$ is the realisation of the simplicial map

$$
\begin{array}{ccc}
\mathbb{Z}^{n} & \longrightarrow & \mathbf{F}^{n} \\
\lambda & \longmapsto & \left(R^{\lambda}, \ldots, R^{\lambda}\right) .
\end{array}
$$

The classifying map $f: S^{1} \times K \longrightarrow B K$ for the bundle $D$ is the realisation of the simplicial map

$$
f: \begin{array}{ccc}
\mathbb{Z}^{n} \times K & \longrightarrow & K^{n} \\
\left(k, \lambda_{1}, \ldots, \lambda_{n}\right) & \longmapsto & \left(k^{\lambda_{1}}, \ldots, k^{\lambda_{n}}\right) .
\end{array}
$$

The homology of $S^{1}$ is the Eilenberg-Mac Lane group homology $H_{*}(\mathbb{Z})$ of $\mathbb{Z}$ (see [10]). Denote by $x$ a generator of $\mathbb{Z}$. A generator of $H_{1}(\mathbb{Z})$ is given by $x$.

Since the centre of $K$ is finite, on the one hand $K$ and $K_{c}$, and on the other hand $B K$ and $B K_{c}$ have naturally isomorphic cohomologies. In addition, every $K$-principal bundle defines a $K_{c}$-principal bundle in a natural way: one just has to compose the classifying map of the $K$-principal bundle with the projection $B K \longrightarrow B K_{c}$. The simplicial version of this last projection is given by the maps $K^{n} \longrightarrow K_{c}^{n}$. Moreover, the characteristic classes of the induced $K_{c}$-principal bundle are the same as the one of the $K$-principal bundle. 
The cohomology of $B K_{c}$ is the cohomology of a double complex $\Omega^{p, q}=$ $\Omega^{p}\left(\mathrm{NK}_{c}(q)\right)$ with two differentials (see [6]): one is the usual differential of forms $\mathrm{d}: \Omega^{p}\left(\mathrm{NK}_{c}(q)\right) \longrightarrow \Omega^{p+1}\left(\mathrm{NK}_{c}(q)\right)$ and the other is

$$
\begin{gathered}
\delta: \Omega^{p}\left(\mathrm{NK}_{c}(q)\right) \longrightarrow \Omega^{p}\left(\mathrm{NK}_{c}(q+1)\right) \\
\delta=\sum_{i=0}^{q+1}(-1)^{i} \varepsilon_{i}^{*}
\end{gathered}
$$

Recall that the maps $\varepsilon_{i}$ were defined in (4). The two differentials $\mathrm{d}$ and $\delta$ commute and the total differential is $\mathrm{d}+(-1)^{p} \delta$ on $\Omega^{p, q}$. Shulman [13] (see also Bott, Shulman and Stasheff [4]) proved that each class $c_{j}$ in $H^{2 r_{j}}\left(B K_{c}\right)$ can be represented by a form $c_{j, 1}+\cdots+c_{j, r}$ in $\Omega^{*, *}$, with $c_{j, k} \in \Omega^{2 r-k, k}$. That this form is closed means that

$$
\begin{aligned}
\mathrm{d} c_{j, 1} & =0 \\
\delta c_{j, k} & =(-1)^{k} \mathrm{~d} c_{j, k+1} \text { for } 1 \leq j<r_{j} \\
\delta c_{j, r} & =0 .
\end{aligned}
$$

Also, the closed form $c_{j, 1} \in \Omega^{2 r_{j}-1}\left(K_{c}\right)$ is a representative of $b_{j}$.

Everything in the above paragraph can be done equivariantly. One just has to replace cohomology by equivariant cohomology, the complex $\Omega^{p, q}=\Omega^{p}\left(\mathrm{NK}_{c}(q)\right)$ by $\Omega_{K}^{p, q}=\Omega_{K}^{p}\left(\mathrm{NK}_{c}(q)\right)$, the differential d by $\mathrm{d}_{K}$, the forms $c_{j}$ by their respective extensions $c_{j}^{K}$ in $H_{K}^{2 r_{j}}\left(B K_{c}\right)$, and so on. Hence it is clear that the pairing $\left\langle x, f^{*} c_{j}\right\rangle$ is $b_{j}^{K}$. This together with the fact that the restriction of $D$ to $S^{1}$ is trivial proves Lemma 3.5.

Proof of Remark 2.5. I chose $c_{j, 1}^{K}$ as a representative $\bar{b}_{j}^{K}$ of $b_{j}^{K}$. The section

$$
\begin{array}{ccc}
s: \quad B K & \longrightarrow & E K \times_{K} K \\
{[u]} & \longmapsto & {[u, 1]}
\end{array}
$$

is obtained from the inclusion of the identity 1 into $K$ by applying the functor, from $K$-spaces to spaces,

$$
X \longmapsto X \times_{K} E K .
$$

Because the inclusion map $\{1\} \longrightarrow K$ is constant and because $c_{j, 1}^{K}$ is of odd degree, it follows that $s^{*} b_{j}^{K}=s^{*} c_{j, 1}^{K}=0$. This proves Remark 2.5.

Proof of Lemma 3.7. The equivariant forms $c_{j, 1}^{K}$ and $c_{j, 2}^{K}$ satisfy $\mathrm{d}_{K} c_{j, 2}^{K}=-\delta c_{j, 1}^{K}$. A classifying map for the $K$-equivariant $K_{c}$-principal bundle $E$ is the realisation of the equivariant simplicial map

$$
\Psi: \begin{array}{ccc}
Y_{\beta} \times \Pi^{n} & \longrightarrow & K_{c}^{n} \\
\left(\rho, p_{1}, \ldots, p_{n}\right) & \longmapsto & \left(\rho\left(p_{1}\right), \ldots, \rho\left(p_{n}\right)\right) .
\end{array}
$$

The homology of $\Sigma$ is the Eilenberg-Mac Lane group homology $H_{*}(\boldsymbol{\Pi})$. A generator of $\mathrm{H}_{2}(\boldsymbol{\Pi})$ is (see [7, Proposition 3.9])

$$
c=\sum_{i=1}^{2 g} \frac{\partial R}{\partial x_{i}} \otimes x_{i},
$$


where $\frac{\partial R}{\partial x_{i}}$ is the Fox free differential calculus (see [7, Section 3]). In fact,

$$
\frac{\partial R}{\partial x_{i}}=\gamma_{j}^{0}-\gamma_{i}^{1}
$$

where $\gamma_{i}^{\tau} \in \mathbf{F}$ is

$$
\begin{array}{ccc}
\gamma_{2 i-1}^{0} & = & \prod_{l=1}^{i-1}\left[x_{2 l-1}, x_{2 l}\right] \\
\gamma_{2 i-1}^{1} & = & \gamma_{2 i-1}^{0} x_{2 i-1} x_{2 i} x_{2 i-1}^{-1} \\
\gamma_{2 i}^{0} & = & \gamma_{2 i-1}^{0} x_{2 i-1} \\
\gamma_{2 i}^{1} & = & \gamma_{2 i-1}^{0}\left[x_{2 i-1}, x_{2 i}\right]
\end{array}
$$

A representative $\bar{a}_{j}^{\prime}$ of $a_{j}^{\prime}$ is given by

$$
\begin{aligned}
\bar{a}_{j}^{\prime} & =\left\langle\sum_{i=1}^{2 g} \frac{\partial R}{\partial x_{i}} \otimes x_{i}, \Psi^{*} c_{j, 2}^{K}\right\rangle \\
& =\sum_{i=1}^{2 g} \sum_{\tau=0,1}(-1)^{\tau}\left\langle\gamma_{i}^{\tau} \otimes x_{i}, \Psi^{*} c_{j, 2}^{K}\right\rangle \\
& =\sum_{i=1}^{2 g} \sum_{\tau=0,1}(-1)^{\tau}\left(\operatorname{ev}_{\gamma_{i}^{\tau}} \times \mathrm{ev}_{x_{i}}\right)^{*} c_{j, 2}^{K},
\end{aligned}
$$

where, for $p \in \mathbf{F}$, the evaluation map is

$$
\begin{array}{ccc}
e v_{p}: \operatorname{Hom}\left(\mathbf{F}, K_{c}\right) & \longrightarrow & K_{c} \\
\rho & \longmapsto & \rho(p) .
\end{array}
$$

The last line of the above computation of $\bar{a}_{j}^{\prime}$ clearly shows that $\bar{a}_{j}^{\prime}$ extends to a form on $K^{2 g}$. The differential of this form is

$$
\begin{aligned}
\mathrm{d} \bar{a}_{j}^{\prime} & =\sum_{i=1}^{2 g} \sum_{\tau=0,1}(-1)^{\tau}\left(\mathrm{ev}_{\gamma_{i}^{\tau}} \times \mathrm{ev}_{x_{i}}\right)^{*} \mathrm{~d} c_{j, 2}^{K} \\
& =-\sum_{i=1}^{2 g} \sum_{\tau=0,1}(-1)^{\tau}\left(\mathrm{ev}_{\gamma_{i}^{\tau}} \times \mathrm{ev}_{x_{i}}\right)^{*} \delta c_{j, 1}^{K} \\
& =-\sum_{i=1}^{2 g} \sum_{\tau=0,1}(-1)^{\tau}\left(\mathrm{ev}_{\gamma_{i}^{\tau}} \times \mathrm{ev}_{x_{i}}\right)^{*} \sum_{l=0}^{2}(-1)^{l} \varepsilon_{l}^{*} c_{j, 1}^{K} \\
& =-\sum_{i=1}^{2 g} \sum_{\tau=0,1} \sum_{l=0}^{2}(-1)^{\tau+l}\left(\varepsilon_{l} \circ\left(\mathrm{ev}_{\gamma_{i}^{\tau}} \times \mathrm{ev}_{x_{i}}\right)\right)^{*} c_{j, 1}^{K} \\
& =\sum_{i=1}^{2 g} \sum_{\tau=0,1} \sum_{l=0}^{2}(-1)^{1+\tau+l} \mathrm{ev}_{z_{i, l}^{\tau}}^{*} c_{j, 1}^{K},
\end{aligned}
$$

where

$$
z_{i, 0}^{\tau}=x_{i}, z_{i, 1}^{\tau}=\gamma_{i}^{\tau} x_{i} \text { and } z_{i, 2}^{\tau}=\gamma_{i}^{\tau}
$$


Because

$$
\begin{aligned}
\mathrm{ev}_{z_{i, 0}^{0}}^{*} & =\mathrm{ev}_{z_{i, 0}^{1}}^{*} \\
\mathrm{ev}_{z_{2 i, 1}^{1}}^{*} & =\mathrm{ev}_{z_{2 i-1,2}^{1}}^{*} \\
\mathrm{ev}_{z_{2 i-1,1}^{0}}^{*} & =\mathrm{ev}_{z_{2 i, 2}^{0}}^{*} \\
\mathrm{ev}_{z_{2 i, 1}^{0}}^{*} & =\mathrm{ev}_{z_{2 i-1,1}^{1}}^{*} \\
\mathrm{ev}_{z_{2 i+1,2}^{0}}^{*} & =\mathrm{ev}_{z_{2 i, 2}^{1}}^{*}
\end{aligned}
$$

many terms cancel each other in the expression (6) above. I am left with

$$
\mathrm{d} \bar{a}_{j}^{\prime}=\mathrm{ev}_{z_{2 g, 2}^{1}}^{*} c_{j, 1}^{K}-e v_{z_{1,2}^{0}}^{*} c_{j, 1}^{K} .
$$

But $z_{1,2}^{0}=1$ the identity of $\mathbf{F}$ and hence $e v_{z_{1,2}^{0}}$ is a constant map, whereas $z_{2 g, 2}^{1}=\prod_{j=1}^{g}\left[x_{2 j-1}, x_{2 j}\right]$ and hence $\mathrm{ev}_{z_{2 g, 2}^{1}}^{*}=\Phi^{*}$. Since $c_{j, 1}^{K}$ is a representative of $b_{j}^{K}$, I deduce that on $K^{2 g}$

$$
\mathrm{d} \bar{a}_{j}^{\prime}=\Phi^{*} \bar{b}_{j}^{K}
$$

This proves Lemma 3.7.

\section{Acknowledgement}

I would like to thank Frances Kirwan for her comments on a preliminary version of this article and Lisa Jeffrey for sending me a copy of Shulman [13]. I also thank the referee for his/her carefull reading of the present paper and for the corrections he/she proposed. I would like to thank Johannes Huebschmann for pointing out to me the similarities between certain constructions I used in the present article and certain contstructions appearing in his article [8].

The author was supported by a Marie-Curie Fellowship, EC Contract Ref: HPMF-CT-2002-01850.

\section{References}

[1] M. F. Atiyah and R. Bott, The Yang-Mills Equations Over Riemann Surfaces, Phil. Trans. Royal Soc. Lond. 308 (1982), 523-615.

[2] A. Alekseev, A. Malkin and E. Meinrenken, - Lie group valued moment maps, J. Differential Geom. 48 (1998), 445-495.

[3] I. Biswas and N. Raghavendra, Canonical generators of the cohomology of moduli of parabolic bundles on curve, Math. Ann. 306 (1996), 1-14.

[4] R. Bott, H. Shulman and J. Stasheff, On the de Rham theory of certain classifying spaces, Adv. Math. 20 (1976), 43-56.

[5] R. Bott, S. Tolman and J. Weitsman, Surjectivity for Hamiltonian Loop Group Spaces, Invent. Math. 155 (2004), 225-251.

[6] J. L. Dupont, Simplicial de Rham cohomology and characteristic classes of flat bundles, Topology 15 (1976), 233-245.

[7] W. Goldman, The symplectic nature of the fundamental groups of surfaces, Adv. Math. 54 (1984), 200-225.

[8] J. Huebschmann, Extended moduli spaces, the Kan construction, and lattice gauge theory, Topology 38 (1999), 555-596. 
[9] L. Jeffrey, Group cohomology construction of the cohomology of moduli spaces of flat connections on 2 manifolds, Duke Math. J. 77 (1995), 407-429.

[10] S. Mac Lane, Homology, Grundlehren Math. Wiss. 114 (1963), Springer-Verlag, Berlin.

[11] S. Racanière, Restriction map in a regular reduction of $\mathbf{S U}(n)^{2 g}$, Comment. Math. Helv. 78 (2003), 394-417.

[12] G. Segal, Classifying spaces and spectral sequences, Inst. Hautes Études Sci. Publ. Math. 34 (1968), 105-112.

[13] H. B. Shulman, Characteristic Classes and Foliations, Doctorate (1968), University of California, Berkeley.

Mathematics Department, South Kensington Campus, Imperial College London, SW7 2AZ, UK

E-mail address: s.racaniere@ic.ac.uk $U R L:$ www.ma.ic.ac.uk/ racani 